\title{
Excluding blood donors at high risk of HIV infection in a west African city
}

\author{
Richard Schutz, Dominique Savarit, Jean-Claude Kadjo, Veronique Batter, N'Valy Kone, \\ Guy La Ruche, Alain Bondurand, Kevin M De Cock
}

Abstract

Objective-To examine the potential impact of deferral of blood donors at high risk of HIV infection in a west African city where blood is screened for HIV antibodies but no other special measures are taken to protect the blood supply.

Design-Cross sectional study.

Setting-National Blood Transfusion Centre and Project RETRO-CI, an international collaborative AIDS research project, Abidjan, Côte d'Ivoire.

Subjects-1257 male first time blood donors.

Interventions-Blood donors were interviewed about demographic and behavioural characteristics and tested for HIV antibodies by enzyme immunoassay and, if positive, synthetic peptide based tests.

Main outcome measures-HIV antibody status in relation to presence of behavioural risk factors; calculation of sensitivity, specificity, and predictive values of specific criteria for excluding HIV infected donors.

Results-The overall prevalence of HIV infection was $\mathbf{1 1 \cdot 4 \%}$. The most important risk factors for HIV positivity were prostitute contact and being aged 30-39 years. For identifying seropositive donors individual criteria had sensitivity, specificity, and positive predictive values ranging from $15 \%$ to $98 \%$, $38 \%$ to $91 \%$, and $17 \%$ to $30 \%$ respectively. Prostitute contact in the past five years would have excluded $31 \%$ of all donors and $73 \%$ of $\mathrm{HIV}$ infected donors. $27 \%$ of those excluded would have been HIV positive.

Conclusions-The widespread assumption that donor deferral is not feasible in sub-Saharan Africa needs reassessment. In Abidjan this approach was well accepted and potentially effective. Donor deferral requires evaluation as a strategy for improving blood safety in resource poor areas with high rates of HIV infection.

Projet RETRO-CI, Abidjan, Côte d'Ivoire Jean-Claude Kadjo, computer operator

Kevin M De Cock, director

Division of HIV/AIDS, National Center for Infectious Diseases, Centers for Disease Control and Prevention, Atlanta, Georgia, USA

Veronique Batter, statistician

Correspondence to: Dr Kevin M De Cock, Department of Clinical Sciences, London School of Hygiene and Tropical Medicine, London

WC1E 7HT.

$B M \mathcal{F} 1993 ; 307: 1517-9$
Although there has been little systematic study, it has been assumed that excluding high risk donors in Africa is not possible because rates of HIV infection in the general population are high $(\mathrm{N}$ Nzila, $\mathrm{R} L$ Colebunders, J M Mann, H Francis, K Nseka, and $\mathrm{J}$ W Curran, paper presented at third international conference on AIDS, Washington, 1987 (abstract W4.6)). ${ }^{10}$ We assessed whether excluding donors likely to be infected with HIV was feasible in a large west African city and what the impact might be on the blood transfusion service in terms of availability of blood.

\section{Subjects and methods}

Abidjan, the largest city in Côte d'Ivoire, has a population of around 2 million. Blood for the city and the surrounding area is provided by the Centre National de Transfusion Sanguine, which in 1990 collected 30165 units. The study was conducted between February and November 1991 and was restricted to first time donors. These made up $42 \%$ of the donor pool, the rest being repeat donors $(52 \%)$ or family members or acquaintances of patients in hospital $(6 \%)$. Because only $13 \%$ of donors were women, this report concerns only men. Blood donation is voluntary.

Interviews with donors-Donors participating in the study were a sample of convenience recruited in a nonselective, arbitrary manner depending on availability of staff. Donors were asked to respond to a verbal questionnaire administered by one of three male clerks who volunteered. The questionnaires concerned basic descriptive and demographic information as well as sexual behaviour, including sex with prostitutes. Having multiple sex partners was defined as having more than one sex partner in the previous 12 months. Having sex with a prostitute was defined as exchanging money or goods for sexual intercourse.

HIV serology-Donated blood was screened for antibodies to HIV-1 and HIV-2 by means of an enzyme immunoassay from one of several manufacturers (Boehringer Mannheim; Diagnostics Pasteur, Paris; Abbott Laboratories, Frankfurt). Samples reactive on screening were tested by one of the other enzyme immunoassays. Repeatedly reactive specimens were further tested by a synthetic peptide based assay (Pepti LAV 1-2, Diagnostics Pasteur)." All units of blood reactive in the first assay were discarded.

Statistical analysis-Data were analysed by computer software packages for epidemiological and statistical studies (EPI-INFO 5.1; SAS 6.04; BMDP (1990)). For analysis no distinction was made between HIV-1, HIV-2, or dual serological reactivity, subjects being grouped as HIV positive or HIV negative. Variables associated with HIV positivity on univariate analysis were assessed in a multivariate analysis by using logistic regression. 


\section{Results}

All men invited to participate agreed. Of the 1257 donors interviewed and tested, $143(11.4 \%)$ were HIV positive. Characteristics of the study population are shown in table I. Most of the donors were born in Côte d'Ivoire, were aged 20-29, and had at least secondary education.

Table II lists the risk factors for HIV infection on univariate analysis. Sixty six (5\%) of the men reported only one sex partner in the previous 12 months and 360 $(29 \%)$ no sex partner. The two thirds $(831 / 1257)$ of men with more than one sex partner in the previous year accounted for $140(98 \%)$ of the $143 \mathrm{HIV}$ infections. HIV seroprevalence rates in men with no sex partner, one partner, or multiple partners in the previous year were nil, $1 \%$, and $17 \%$ respectively $(\mathrm{p}<0.001)$

Risk factors significantly associated with HIV infection included multiple sex partners, a history of sex with prostitutes, a history of sexually transmitted diseases, age 20 or older, and being illiterate. Being celibate protected against HIV infection $(p<0.01)$.

In the multivariate analysis sex with prostitutes in the past five years without regular use of condoms was the strongest risk factor for HIV infection (odds ratio $10.0 ; 95 \%$ confidence interval 6.6 to $15 \cdot 0$ ). Once this factor was included in the model, being aged 30-39 remained the only variable significantly associated (odds ratio $6.9 ; 2.6$ to 18.0 ). Prostitute contact in the past five years (irrespective of condom use) was almos as strong a risk factor in the multivariate model (odds ratio $8.4 ; 5.6$ to $12 \cdot 6$ ) as prostitute contact without regular condom use.

Table III gives the sensitivity, specificity, and positive and negative predictive values for individual criteria for determining whether a donor was infected with HIV and the proportion of all donors who would have been excluded. Individual criteria had sensitivity values ranging from $15 \%$ to $98 \%$ for identifying seropositive donors, specificity ranging from $38 \%$ to $91 \%$. Positive predictive value-that is, the proportion

Median age of subjects was 25 Age range was 16-60. of donors responding positively to a criterion and who were infected with HIV-ranged from $17 \%$ to $30 \%$. Prostitute contact in the past five years without regular condom use was associated with a sensitivity of $72 \%$ and the highest positive predictive value recorded $(30 \%)$. Rates of sensitivity and positive predictive value for prostitute contact in the past five years irrespective of condom use were $73 \%$ and $27 \%$ respectively. Either criterion would have excluded fewer than one third of all donors.

\section{Discussion}

In Zaire Nzila et al (third international conference on AIDS, Washington, 1987 (abstract W4.6)) found no clinical differences between HIV positive and HIV negative donors, leading to the widespread assumption that excluding donors at high risk of HIV infection was unfeasible in Africa. ${ }^{10}$ Though HIV testing of donated blood is the rule in most major African cities, deferral of high risk donors has not been adopted to any significant degree. This study is one of the first attempts to examine systematically the potential impact of such an intervention.

As shown in other groups in Abidjan ${ }^{12}$ and elsewhere in Africa, ${ }^{13}$ blood donors with HIV infection had certain well defined risk factors. Selected characteristics strongly associated with HIV seropositivity included contact with women prostitutes, especially without regular condom use, a history of sexually transmitted diseases, and age. Thus even in areas with high rates of HIV infection not everyone is at equal risk. It should therefore be feasible to identify those most likely to be HIV positive and exclude them from donating blood.

A history of contact with prostitutes in the past five years identified $73 \%$ of infected donors (104/143; sensitivity $73 \%$ ) and was absent in $75 \%$ of HIV negative donors (834/1114; specificity $75 \%$ ). Of all donors with this history, $27 \%(104 / 384)$ were HIV infected (positive predictive value $27 \%$ ). Applying this criterion would have resulted in $31 \%$ of all units being discarded (table III). Thus at a cost of less than one third of the total units of blood donated almost three quarters of infected units could have been excluded, and over a quarter of units excluded would have been from donors who were infected with HIV. Using more complicated criteria for donor exclusion-for example, prostitute contact in the past five years or age $30-39$, or both-was more sensitive for identifying infected donors but would have resulted in over half the donors being rejected.

There are at least three advantages of excluding high risk donors for blood transfusion services in a developing country. Firstly, less of scarce resources will be spent on supplemental testing or other interventions for HIV positive subjects; donors at high risk of infection could be referred to the appropriate services for testing, counselling, and follow up. Secondly,

TABLE III-Effects of excluding blood donors according to specific criteria. Results expressed as numbers (\%) of subjects

\begin{tabular}{|c|c|c|c|c|c|}
\hline & $\begin{array}{l}\text { Sensitivity } \\
\text { (proportion of HIV } \\
\text { positive donors } \\
\text { excluded) }\end{array}$ & $\begin{array}{l}\text { Specificity } \\
\text { (proportion of HIV } \\
\text { negative donors not } \\
\text { excluded) }\end{array}$ & $\begin{array}{l}\text { Positive predictive } \\
\text { value (proportion of } \\
\text { donors excluded } \\
\text { who were HIV } \\
\text { positive) }\end{array}$ & $\begin{array}{l}\text { Negative predictive } \\
\text { value (proportion of } \\
\text { donors not excluded } \\
\text { who were HIV } \\
\text { negative) }\end{array}$ & All excluded \\
\hline \multicolumn{6}{|l|}{ More than one sex partner in past } \\
\hline 12 months & $140 / 143(98)$ & $423 / 1114(38)$ & $140 / 831(17)$ & $423 / 426(99)$ & $831 / 1257(66)$ \\
\hline Prostitute contact ever & $120 / 143(84)$ & $617 / 1114(55)$ & $120 / 617(19)$ & $617 / 640(96)$ & $617 / 1257(49)$ \\
\hline Prostitute contact in past five years & $104 / 143(73)$ & $834 / 1114(75)$ & $104 / 384(27)$ & $834 / 873(96)$ & $384 / 1257(31)$ \\
\hline \multicolumn{6}{|l|}{ Prostitute contact in past five years } \\
\hline without regular use of condoms & $103 / 143(72)$ & $874 / 1114(78)$ & $103 / 343(30)$ & $874 / 914(96)$ & $343 / 1257(27)$ \\
\hline \multicolumn{6}{|l|}{ Several sexually transmitted diseases } \\
\hline in past five years & $39 / 143(27)$ & $943 / 1114(85)$ & $39 / 210(19)$ & $943 / 1047(90)$ & $210 / 1257(17)$ \\
\hline Illiterate & $21 / 143(15)$ & $1019 / 1114(91)$ & $21 / 116(18)$ & $1019 / 1141(89)$ & $116 / 1257(9)$ \\
\hline Age 30-39 & $51 / 143(36)$ & $931 / 1114(84)$ & $51 / 234(22)$ & $931 / 1023(91)$ & $234 / 1257(19)$ \\
\hline \multicolumn{6}{|l|}{ Prostitute contact in past five years } \\
\hline or aged 30-39 & $123 / 143(86)$ & $690 / 1114(62)$ & 1231347 (22) & $0901610(91)$ & $34 / 11257(44)$ \\
\hline
\end{tabular}




\section{Clinical implications}

- HIV infected blood may still be transfused in Africa because of lack of access to HIV testing, lack of adequate laboratory quality control, and from donors being in the seroconversion period

- Donor deferral has not been thought feasible in Africa because heterosexual transmission accounts for most cases of HIV infection, making risk groups difficult to identify

- Not everyone is at equal risk for HIV infection; in Abidjan, Côte d'Ivoire, important risk factors for HIV infection among male blood donors were prostitute contact and age

- Behavioural characteristics identified a large proportion of donors who were most likely to be HIV infected; a history of prostitute contact in the past five years identified $73 \%$ of $\mathrm{HIV}$ infected donors while being present in $31 \%$ of all donors

- Excluding donors based on behavioural characteristics of those most at risk of HIV infection is feasible in Abidjan. Donor deferral merits evaluation as an intervention to improve blood safety in developing countries
Although the priority must remain to extend HIV testing to all units of blood transfused, donor deferral merits investigation in resource poor areas. Exclusion criteria may differ between societies, and this study should be repeated in other developing countries. Additional requirements for improved blood safety in the developing world are country specific guidelines and supervision of transfusion practices and more emphasis on eliminating all but lifesaving transfusions.

1 Doll LS, Petersen LR, White CR, Ward JW, HIV Blood Donor Study Group. Human immunodeficiency virus type 1 infected blood donors: behavioral characteristics and reasons for donation. Transfusion 1991;31:704-9.

2 Mann J, Tarantola DJM, Netter TW, eds. Blood safety and blood products. In: AIDS in the world. Cambridge, Mass: Harvard University Press, 1992:421-37.

3 Chin J, Mann JM. Global surveillance and forecasting of AIDS. Bull World Health Organ 1989;67:1-7.

4 Greenberg AE, Nguyen-Dinh P, Mann JM, Kabote N, Colebunders RL, Francis $\mathrm{H}$, et al. The association between malaria, blood transfusions and HIV seropositivity in a pediatric population in Kinshasa, Zaire. $J A M A$ 1988;259:545-9.

5 Lackriz EM, Campbell CC, Ruebush TK II, Hightower AW, Wakube W, Steketee RW, et al. Effect of blood transfusion on survival among children in a Kenyan hospital. Lancet 1992;340:524-8.

6 Jager $\mathrm{H}$, Jersiid $\mathrm{C}$, Emmanuel JC. Safe blood transfusions in Africa. AIDS 1991; 5:S163-8.

7 N'tita I, Mulanga K, Dulat C, Lusamba D, Rehle H, Korte R, et al. Risk of transfusion-associated HIV infection in Kinshasa, Zaire. AIDS 1991;5: 437-41.

serological testing cannot identify donors in the seroconversion period; exclusion on risk factors may be the only way to deal with what could be a common finding in areas of high incidence of HIV infection.' Lastly, laboratory errors are inevitable, and false negative results are less likely when HIV prevalence in the donor pool is low.

The absence of donor exclusion before the introduction of HIV antibody testing may in part explain the greater number of HIV infected transfusion recipients in France than in other European countries. ${ }^{14}$ Even with blood screening, the current risk of transfusion transmitted HIV infection in Abidjan-and presumably in other African cities with high HIV infection incidence and prevalence-exceeds that which existed in industralised countries at the beginning of the HIV epidemic. ${ }^{9}$
MRC Environmental

Epidemiology Unit,

University of

Southampton,

Southampton General

Hospital, Southampton

SO9 4XY

C Osmond, statistician

D J P Barker, director

P D Winter, computing manager

C H D Fall, paediatrician

S J Simmonds, research

assistant

Correspondence to:

Professor Barker.

$B M 7$ 1993;307:1519-24

\section{Early growth and death from cardiovascular disease in women}

\section{Osmond, D J P Barker, P D Winter, C H D Fall, S J Simmonds}

\begin{abstract}
Objective-To determine whether the link suggested between growth in utero and during infancy and death from cardiovascular disease in men is also present in women.

Design-Follow up study of women and men whose birth weight and weight at 1 year of age had been recorded.

Setting-Hertfordshire, England.

Subjects-5585 women and 10141 men born during 1911-30.

Main outcome measures-Standardised mortality ratios for cardiovascular disease.

Results-Among women and men death rates from cardiovascular disease fell progressively between the low and high birth weights groups $\left(\chi^{2}=4.3, p=0.04\right.$ for women, $\chi^{2}=8.5, p<0.005$ for men). Cardiovascular deaths in men but not women were also strongly related to weight at 1 year, falling progressively between the low and high weight groups $\left(\chi^{2}=27.5, p<0.0001\right)$. The highest cardiovascular death rates in women were among those with below average birth weight but above average weight at 1 year. In men the highest rates were among those with below average birth weight and below average weight at 1 year.

Conclusion-Relations between cardiovascular
\end{abstract}

disease and birth weight are similar in men and women. In men cardiovascular disease is also related to weight gain in infancy.

\section{Introduction}

The first direct evidence that coronary heart disease may originate in utero and during infancy came from a study of men born in Hertfordshire, England.' The birth weight and weight at 1 year of all babies born in the county since 1911 had been recorded. Among 5654 men born during 1911-30, death rates from coronary heart disease fell progressively between those with the lowest and highest weights at 1 year. There were similar, though less strong, trends with birth weight. A study of 1586 men born in Sheffield similarly showed that death rates from cardiovascular disease fell progressively between those who had low and high birth weight. ${ }^{2}$ This trend did not depend on differences in the length of gestation and therefore reflected differences in fetal growth rates.

Subsequent studies suggested that growth in utero and during infancy and the method of infant feeding were linked to the main risk factors for cardiovascular disease. Men and women who had had reduced rates of fetal growth or low weights at 1 year had raised prevalences of hypertension and non-insulin dependent 